\title{
From acute to chronic pain after thoracic surgery: the significance of different components of the acute pain response
}

This article was published in the following Dove Press journal: Journal of Pain Research

\author{
Morten Rune \\ Blichfeldt-Eckhardt ${ }^{1,2}$ \\ Claus Andersen ${ }^{2}$ \\ Helle Ørding' \\ Peter B Licht ${ }^{3}$ \\ Palle Toft ${ }^{2}$ \\ 'Department of Anesthesiology, Vejle \\ Hospital, Vejle, Denmark; '2Department \\ of Anesthesiology and Intensive Care, \\ Odense University Hospital, Odense, \\ Denmark; ${ }^{3}$ Department of Thoracic \\ Surgery, Odense University Hospital, \\ Odense, Denmark
}

Purpose: Acute postoperative pain is a strong predictor for postthoracotomy pain syndrome (PTPS), but the mechanism is unknown. Even though thoracic pain is usually considered the dominating acute pain after thoracic surgery, up to $45 \%$ of patients consider shoulder pain to be dominating pain and often this shoulder pain is referred visceral pain. This study aims to examine which components of the acute pain response after thoracic surgery were associated with PTPS and if any signs of a generalized central hypersensitivity could be identified in patients with PTPS.

Patients and methods: In a prospective cohort study, 60 consecutive patients for lobectomy were included and examined preoperatively and 12 months postoperatively for pain and signs of hypersensitivity using a comprehensive protocol for quantitative sensory testing. Thoracic pain, shoulder pain, referred pain, and overall pain were assessed five times daily during the first four postoperative days.

Results: Sixteen patients ( $31 \%$ of the 52 patients who completed the study) developed PTPS. Thoracic pain was the only pain component that was associated with PTPS and was a stronger predictor for PTPS than overall pain. There were no signs of hypersensitivity before or after the operation in patients with PTPS, but patients with PTPS more often suffered from preoperative pain.

Conclusion: Thoracic pain was the only component of the acute pain response that predicted PTPS and was a stronger predictor than overall pain.

Keywords: postthoracotomy pain syndrome, acute postoperative pain, chronic postoperative pain, referred pain, thoracic surgery

\section{Plain language summary}

Chronic pain after chest surgery is a serious and frequent complication affecting $21-61 \%$ of operated individuals. The cause is unclear, but people who suffer from severe pain during the first few days after the operation, more often suffer from chronic pain. Again the reason remains unclear and it is unclear which types of pain are associated with chronic pain. Possibly severe pain, by itself, can induce changes in the nervous system that produce chronic pain. Another possibility is that certain types of damage (ie, on nerves) produce both acute and chronic pain.

We wanted to investigate which types of pain during the first few days (mainly shoulder pain and chest pain) were more likely to be associated with chronic pain.

We included 60 people who had major chest surgery and examined them before the operation, the first 4 days after the operation, and after 12 months. We performed tests of pain thresholds, assessed intensity, and type of pain the first 4 days after the operation and again 12 months after the operation.
Correspondence: Morten Rune Blichfeldt-Eckhardt Intensive Care, Odense University Hospital, Sdr. Boulevard 29, DK-5000 Odense C, Denmark

Tel $+45654|472|$

Fax +456613415

Email morten.rune.blichfeldt-eckhardt@ rsyd.dk 
We found that there was a clear association between pain at the surgical site and the development of chronic pain 12 months after surgery, but no association with shoulder pain or overall pain. There were no changes in pain thresholds before surgery or after surgery.

These results demonstrate that chronic pain is mainly associated with severe wound pain in the first few days after the operation, whereas other kinds of pain are less important.

\section{Introduction}

Postthoracotomy pain syndrome (PTPS) is a serious and frequent complication after thoracic surgery. It affects $21-61 \%$ of patients ${ }^{1-5}$ and reduces activities of daily living in up to $60 \%$ of patients, ${ }^{6,7}$ yet the cause of PTPS has not been established. ${ }^{4,8}$ Though conflicting evidence exists, sex, age, and preoperative pain have been identified as possible predictive factors. As in several other chronic postsurgical pain syndromes, ${ }^{10-12}$ acute postoperative pain is one of the strongest predictors of PTPS, ${ }^{13-16}$ but the mechanism remains unclear. Intraoperative nerve damage has been suggested as a cause of both acute and chronic postoperative pain ${ }^{4,17-20}$; however, several studies have indicated that nerve damage is only part of the cause for PTPS. ${ }^{21-25}$ Another possibility is that the intense postoperative pain causes plastic changes in the central nervous system, leading to central sensitization. ${ }^{8,22,26}$

The acute pain response after thoracic surgery primarily consists of thoracic pain and shoulder pain ipsilateral to the operation. While thoracic pain is usually considered the dominating pain, shoulder pain is described as the dominating pain problem in up to $45 \%$ of patients. ${ }^{27}$ Thoracic pain is believed to be partly somatic and partly neuropathic, whereas shoulder pain is believed to be partly somatic and partly referred visceral pain from the mediastinal and diaphragmatic pleura. ${ }^{28-30}$ It is well known that different types of pain have different implications for the nervous system, and referred pain has been suggested to be a sign of neuronal plasticity and thus an indicator of chronic pain. ${ }^{31}$

Whether some components of the acute pain response after thoracic surgery are more important than others in predicting PTPS has not previously been reported.

Thus, the aim of this study was to examine which components of the acute pain response after thoracic surgery were associated with PTPS and if any signs of a general central hypersensitivity could be identified in patients with PTPS before or after the operation. We hypothesized that both acute thoracic and referred pain would predict PTPS.

\section{Patients and methods}

\section{Patients}

This prospective, observational cohort study was conducted in accordance with the Helsinki Declaration and was approved by the Regional Committees on Health Research Ethics for Southern Denmark (S-20110167) and The Danish Data Protection Agency. Informed, written consent was obtained from all the patients.

Patients scheduled for elective lobectomy at Odense University Hospital, Denmark, who were at least 18 years old and who could speak and read Danish were included between January and December 2012. Patients were excluded if they were reoperated during the first postoperative week, had undergone previous thoracic surgery, suffered from preoperative shoulder pain, any disease in the central nervous system, or peripheral sensory disturbances in the upper extremities. Other results from this cohort have previously been published. ${ }^{27}$

\section{Anesthesia and surgery}

All patients received $1 \mathrm{~g}$ of paracetamol as premedication. Before induction of general anesthesia, a midthoracic epidural was inserted under local anesthesia and patients received a $3 \mathrm{~mL}$ epidural test dose of $2 \%$ lidocaine with $5 \mu \mathrm{g} / \mathrm{mL}$ epinephrine. General anesthesia was induced and maintained with propofol, supported by remifentanil and an epidural infusion of bupivacaine $2.5 \mathrm{mg} / \mathrm{mL}$ with sufentanil $1 \mu \mathrm{g} / \mathrm{mL}$ as intraoperative analgesia. Neuromuscular block was achieved with cisatracurium and a double lumen endobronchial tube was inserted for lung separation ventilation. Before wake up, neuromuscular block was reversed with glycopyrron/neostigmine and $30 \mathrm{mg}$ of keterolac was administered.

Before surgery, all patients were placed in the lateral decubitus position. The surgical procedure was either standard posterolateral thoracotomy (not muscle sparing) or video-assisted thoracic surgery ${ }^{32}$ depending on the surgeon's preference. Following surgical resection, all patients received a single chest tube $\mathrm{Ch} 24$.

Postoperatively, the epidural infusion was changed to bupivacaine $1 \mathrm{mg} / \mathrm{mL}$ with fentanyl $2 \mu \mathrm{g} / \mathrm{mL}$ and adrenalin $0.2 \mu \mathrm{g} / \mathrm{mL}$ for analgesia. Rescue analgesics were ibuprofen or lornoxicam in case of shoulder pain, and intravenous morphine, fentanyl, ketobemidon, or alfentanil as rescue opioids. Epidural infusion was paused upon removal of the chest tube and replaced with slow release morphine. 


\section{Postoperative observations}

Postoperative pain in the thorax (defined as pain in the thorax), shoulder (defined as pain in the shoulder), and overall pain (defined as any pain regardless of location) was assessed during rest and activity on an 11-point numerical rating scale (NRS) $(0=$ no pain, $10=$ worst imaginable pain). Thoracic pain during activity was defined as pain when coughing. Shoulder pain during activity was defined as shoulder pain with $90^{\circ}$ abduction of the shoulder ipsilateral to the operation. Overall pain during activity was defined as worst pain at any site during any activity. On the day of the operation (postoperative day 0 ), pain was assessed at 2, 4, and 6 hours after surgery and on postoperative day (POD) $1-3$ at 6,10 , 14,18 , and 22 o'clock.

At 8 am on POD 1, all patients were examined by the same investigator to characterize possible shoulder pain. The shoulder area and related muscles were palpated thoroughly to find signs of muscle tenderness. Shoulder pain was classified as referred if there were no signs of mechanically triggered shoulder pain which included the following: no muscle tenderness in the shoulder pain area, no shoulder pain could be reproduced by palpating tender muscles in other areas, and shoulder pain was not affected on movement of the shoulder.

At 12 months, all patients were invited to a follow-up visit for evaluation of pain, hospital anxiety and depression scale (HADS), and quantitative sensory testing (QST). Patients were asked about pain in the thorax, shoulder, or other places and whether this pain had lasted since the operation. Pain was rated on the NRS as the maximum and average pain on each location during the last week. Furthermore, patients were asked about relapse, adjuvant therapy (chemotherapy, radiotherapy), and analgesic treatment. Patients who could not appear at a follow-up visit were interviewed over the telephone. All patients with malignant disease were followed up with computed tomography (CT) scans of the thorax 12 months after surgery. Data on CT scans were extracted from patient records.

\section{Psychological testing and preoperative pain}

Preoperatively and 12 months postoperatively, HADS was used to screen for preoperative depression and anxiety symptoms. Cutoff $\geq 8$ for possible anxiety and depression. ${ }^{33}$ All patients were asked about any preoperative pain conditions.

\section{QST}

QST was done preoperatively and after 12 months. One investigator (MRB-E) examined all patients who were in a relaxed supine position at room temperature between $22^{\circ} \mathrm{C}$ and $24^{\circ} \mathrm{C}$. Each test was thoroughly explained and demonstrated on the thenar of the surgical side before testing. Test areas were the thenar contralateral to the surgical side and both shoulders.

\section{Thermal testing}

Cold detection threshold, warm detection threshold, cold pain threshold, and heat pain threshold stimuli were tested using an MSA Thermotester (Somedic AB, Norra Mellby, Sweden). Thresholds were obtained with ramped stimuli $\left(1^{\circ} \mathrm{C} / \mathrm{s}\right)$, which were terminated when the patients pushed a button. Baseline temperature was $32^{\circ} \mathrm{C}$ and cut-off points were $6^{\circ} \mathrm{C}$ and $50^{\circ} \mathrm{C}$. The final threshold was calculated as the mean of three consecutive measurements. ${ }^{34}$

\section{Mechanical testing}

Mechanical detection threshold was tested using modified von Frey hairs (Somedic AB) exerting forces between 5 and $178 \mathrm{~g} / \mathrm{mm}^{2}$. Using the method of limits, the geometric mean was calculated between five series of ascending and descending stimuli intensities as the final threshold. Mechanical pain threshold was tested using a set of seven custom-made weighted pinprick stimulators (flat contact area of $0.2 \mathrm{~mm}$ diameter) that exert forces between 8 and $512 \mathrm{mN}$ (MRC Systems, Heidelberg, Germany). Again, the final threshold was the geometric mean of five series of ascending and descending stimuli intensities. Mechanical pain sensitivity (MPS) was assessed using the same set of pinprick stimulators. For each stimulus, patients were asked to give a pain rating on a 0-100 numerical scale ("0" indicating "no pain," and "100" indicating "most intense pain imaginable"). Each stimulator was tested 5 times in 5 series of 7 randomly delivered stimuli, a total of 35 stimuli. MPS was calculated as the geometrical mean of all numerical pain ratings. ${ }^{34}$

\section{Wind-up ratio}

Wind-up ratio was tested using a weighted pinprick stimulator of $256 \mathrm{mN}$. A single pinprick was compared with a train of 10 stimuli of the same force repeated at a $1 / \mathrm{s}$ rate and given within an area of $1 \mathrm{~cm}^{2}$. Alternating between a single and a train of 10 pinprick stimuli, the test was repeated five times at five different skin sites on the thenar. The patient was asked to give a pain rating on an NRS between 0 and 100 for each single and train of 10 stimuli. $^{34}$

\section{Vibration}

Vibration threshold (VT) test was performed with a vibrameter IV (Somedic $\mathrm{AB}$ ) that was placed over the second 
metacarpal bone on the hand and the nearest bony prominence of RPA (rib). VT was determined as the mean of three series of ascending and descending stimulus intensities.

\section{Pressure}

Pressure pain threshold was performed with an Algometer II (Somedic AB) with a probe area of $1 \mathrm{~cm}^{2}$ and determined as the mean response of three independent measurements, each applied as a slowly increasing ramp of $50 \mathrm{kPa} / \mathrm{s}^{34}$

\section{Statistical analyses}

Because this was an observational study, and because we were unable to find published data on the association between components of the acute pain response and chronic pain after thoracic surgery, a formal study power calculation could not be conducted.

Continuous parametric data are presented as mean with SD. Continuous nonparametric data are presented as medians with 25-75 interquartile ranges and compared using the Mann-Whitney $U$-test. Categorical data are presented as numbers and proportions and analyzed using the $\chi^{2}$ or Fisher's exact test where relevant. A daily mean NRS score was calculated from all five NRS scores during the day. The area under the curve was calculated by cumulating the four daily pain scores for each pain component into one total pain score (TPS) for each pain component. Logistic regression was used to test for association between referred pain and PTPS. Data were analyzed per protocol, and a two-sided $p$-value $<0.05$ was considered statistically significant.

\section{Results}

Overall, 60 patients were included between January and December 2012 and 7 patients were lost to follow-up as specified in Figure 1. Of the 17 patients, who complained of chronic pain in the thorax 12 months after surgery, 1 patient had tumor ingrowth to the chest wall and was excluded from the analysis.

Table 1 shows the demographic and perioperative data. Patients with PTPS were significantly younger and suffered more often from preoperative pain conditions.

\section{Acute pain response vs. chronic pain}

Figure $2 \mathrm{~A}-\mathrm{C}$ shows the daily average pain scores during activity for patients with and without PTPS 12 months

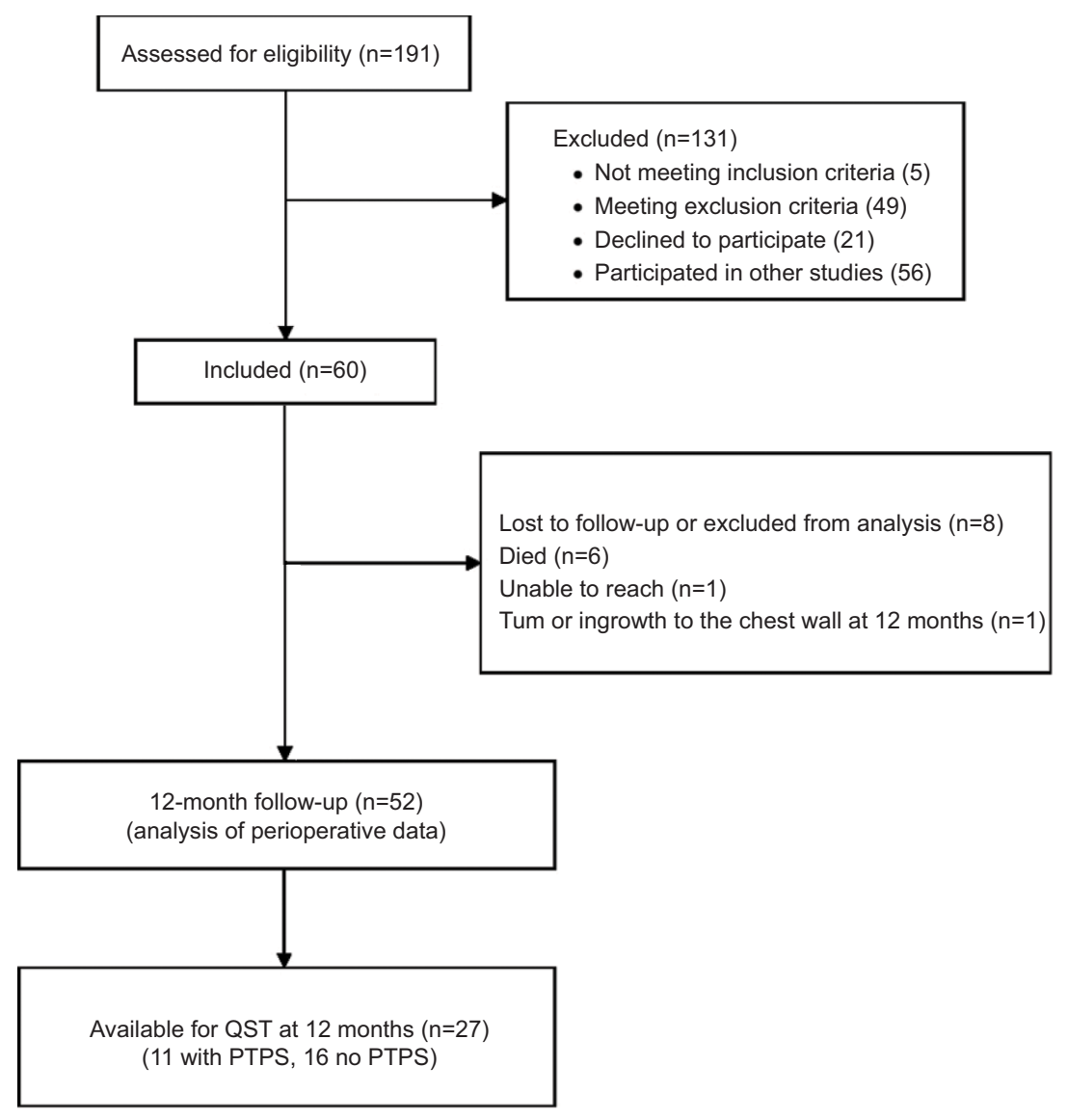

Figure I Flowchart for inclusion and follow-up in the study.

Abbreviations: PTPS, postthoracotomy pain syndrome; QST, quantitative sensory testing. 
Table I Demographic and intraoperative factors

\begin{tabular}{llll}
\hline Variables & PTPS, $\mathbf{n = 1 6}$ & No PTPS, $\mathbf{n = 3 6}$ & p-value \\
\hline Sex, female & $10(63 \%)$ & $19(53 \%)$ & 0.56 \\
Age, years & $60.6(14.9)$ & $67.3(8.5)$ & 0.04 \\
BMI, kg/m & $24.3(4.8)$ & $24.6(4.5)$ & 0.87 \\
FEVI, \% of expected & $86.3(2.3)$ & $85.0(16.3)$ & 0.82 \\
Preoperative chronic pain condition & $8(50 \%)$ & $7(19 \%)$ & 0.03 \\
Preoperative anxiety & $6(38 \%)$ & $9(25 \%)$ & 0.30 \\
Preoperative depression & $4(25 \%)$ & $4(11 \%)$ & 0.22 \\
Thoracotomy: VATS & $12(75 \%): 4(25 \%)$ & $20(56 \%): 16(44 \%)$ & 0.23 \\
Duration of surgery, minutes & $109.6(26.9)$ & $109.4(32.1)$ & 0.98 \\
Days with chest tube & $2.9(3.2)$ & $2.5(2.9)$ & 0.65 \\
Cumulated opioid consumption days I-4, mg morphine equivalents & $142(106)$ & $103(68)$ & 0.12 \\
Postoperative chemotherapy & $6(38 \%)$ & $15(42 \%)$ & 0.08 \\
Postoperative radiotherapy & $2(13 \%)$ & $2(6 \%)$ & 0.75 \\
Relapse of neoplastic disease (\%) & $6(38 \%)$ & $6(17 \%)$ & 0.10
\end{tabular}

Notes: Data are numbers (\%) (chi-squared or Fisher's exact test) or mean (SD) (Student's t-test).

Abbreviations: BMI, body mass index; FEVI, forced expiratory volume in I second; PTPS, postthoracotomy pain syndrome; VATS, video-assisted thoracic surgery.
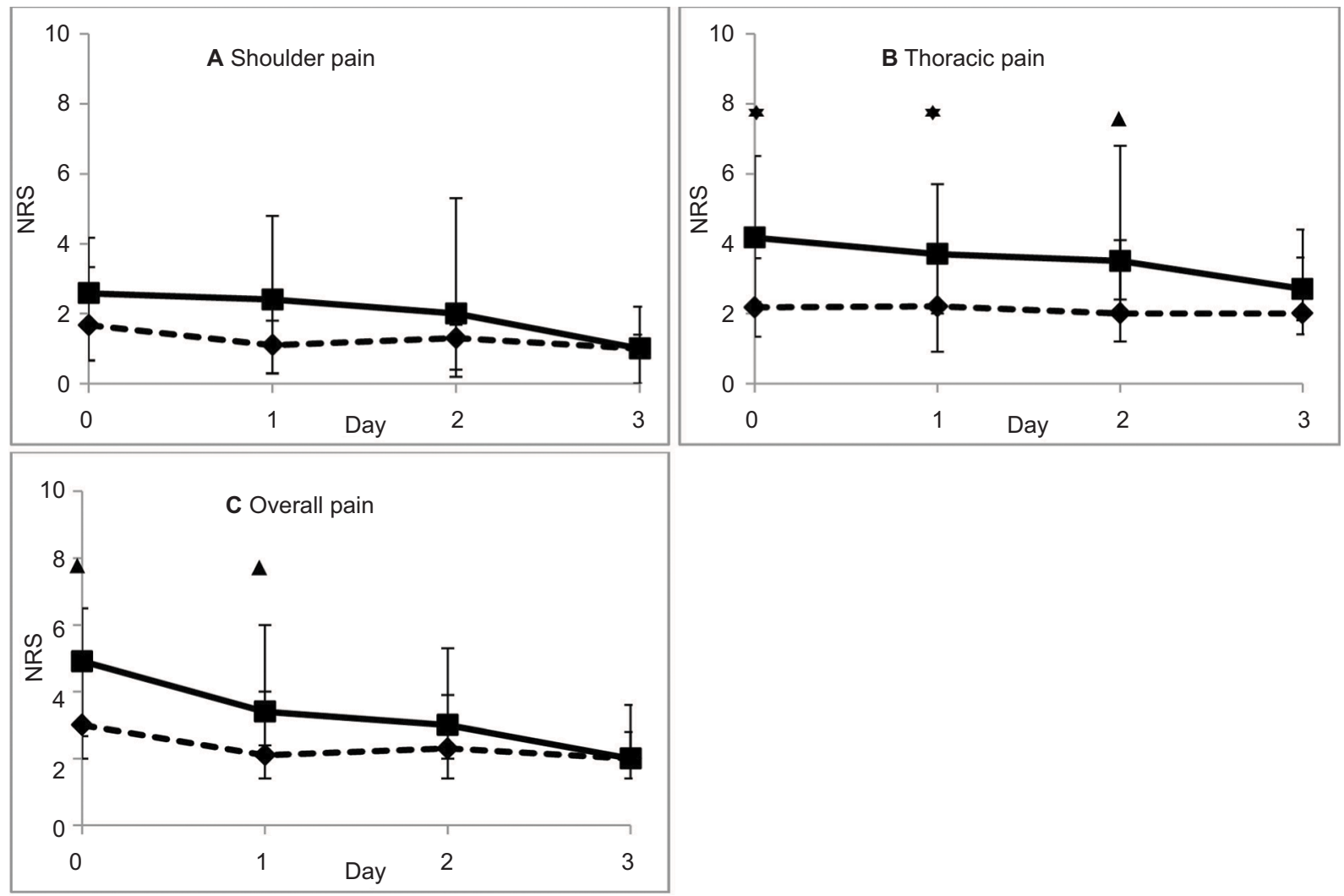

Figure 2 Daily average NRS scores from postoperative days $0-3$.

Notes: - - Postthoracotomy pain syndrome; $\cdot \bullet-\cdot$ no postthoracotomy pain syndrome; ${ }^{*} p<0.0$ I, $\boldsymbol{\Delta} p<0.05$ (Mann-Whitney U-test). Data are presented as medians with 25 and 75 percentiles.

Abbreviation: NRS, numerical rating scale.

after surgery. Most pronounced was the difference in thoracic pain, where PTPS patients experienced significantly more thoracic pain on days $0-2(p=0.004, p=0.009$, and $p=0.045$, respectively), whereas overall pain was only significantly different on days $0-1$ ( $p=0.047$ and $p=0.032$, respectively). There were no significant differences in shoulder pain and no difference in referred pain component (not shown).

Total cumulated pain scores (analog to the area under the curve) for shoulder pain, chest pain, and overall pain are presented in Table 2. Only TPS for thoracic pain was significantly different between the two groups. 


\section{QST}

QST was conducted on all patients preoperatively and 27 patients agreed to participate in the QST examination 12 months postoperatively. There was no difference in any sensory parameters between patients with and without PTPS before or after the operation (Table 3). Thus, there were no signs of generalized hypersensitivity in PTPS patients, and preoperative pain thresholds were not predictive of postoperative pain.

\section{Discussion}

The main finding in this study was that thoracic pain was the only component of the acute pain response that was significantly associated with PTPS, whereas shoulder pain and referred pain were not. Furthermore, only the TPS for thoracic pain was significantly associated with chronic pain, whereas TPS for overall pain was not. In addition, we found no difference in pain thresholds before or after surgery.

The association between the acute pain response and chronic postsurgical pain has been demonstrated in several studies for both thoracic and other types of surgery. ${ }^{10,12-16}$ We believe that this is the first study to address the influence of the individual components of the acute pain response on PTPS and it is noteworthy that our results dispute a generally

Table 2 Total cumulated pain scores

\begin{tabular}{llll}
\hline & $\begin{array}{l}\text { PTPS } \\
(\mathbf{n = 1 6 )}\end{array}$ & $\begin{array}{l}\text { No PTPS } \\
(\mathbf{n = 3 6 )}\end{array}$ & -value \\
\hline TPS-shoulder pain & 7.5 & 4.7 & 0.37 \\
TPS-thoracic pain & 13.6 & 8.8 & 0.03 \\
TPS-overall pain & 12.4 & 10.2 & 0.18 \\
\hline
\end{tabular}

Notes: Data are presented as medians with 25 and 75 percentiles. $p$-Values were compared with the Mann-Whitney U-test.

Abbreviations: PTPS, postthoracotomy pain syndrome; TPS, total pain score, calculated as the cumulated daily mean numerical rating scale scores during activity. accepted theory that acute postoperative pain per se is predictive of chronic postsurgical pain. ${ }^{8,26}$ Instead, it appears that only pain directly related to surgical injury predicts chronic pain. First, this demonstrates that it is important to consider how pain is estimated in future studies of PTPS as not only intensity but also location of postoperative pain could be important as predictors of PTPS. Second, this supports the theory that acute and chronic postoperative pains are correlated, because both are the result of the surgical injury (ie, nerve damage) rather than the theory that acute postoperative pain in itself causes PTPS. This speculation is supported by the finding that only TPS of thoracic pain was associated with PTPS, but this should be tested in a larger study.

We are aware of only one previous study that investigated the relation between components of the acute postoperative pain response and chronic pain. ${ }^{35}$ In this study of patients who underwent cholecystectomy, cumulated visceral pain during the first week was the only part of the acute pain response that was significantly associated with chronic pain following 12 months. These results support our findings that postoperative pain per se is not necessarily predictive of chronic postsurgical pain.

We used a validated comprehensive QST protocol but did not find signs of generalized hypersensitivity in our patients before or after the operation that could otherwise have indicated generalized central sensitization. Preoperative central sensitization has been suggested as a cause for increased acute and chronic postoperative pain, and postoperative central sensitization, triggered by the surgical injury, has been suggested as pathophysiological mechanism for chronic postsurgical pain. Our results, however, are compatible with previous results from two cross-sectional studies that also investigated the patients with PTPS..$^{22,23}$ They found signs of local nerve damage near the surgical scar but did not detect

Table $3 \Delta$ QST (preoperative-postoperative values)

\begin{tabular}{|c|c|c|c|}
\hline Variables & PTPS $(n=I I)$ & No PTPS $(n=16)$ & $p$-value \\
\hline$\Delta \mathrm{CPT},{ }^{\circ} \mathrm{C}$ & $-0.22(-0.57$ to 0.22$)$ & $0.06(-0.4$ to 0.37$)$ & 0.52 \\
\hline$\Delta \mathrm{WDT},{ }^{\circ} \mathrm{C}$ & $0.19(-0.96$ to 0.39$)$ & $0.01(-1$ to 0.64$)$ & 0.94 \\
\hline$\Delta \mathrm{CPT},{ }^{\circ} \mathrm{C}$ & $0.87(-2.54$ to 3.52$)$ & $-0.27(-5.97$ to 3.26$)$ & 0.61 \\
\hline$\Delta \mathrm{HPT},{ }^{\circ} \mathrm{C}$ & $-0.15(-3.52$ to 1.95$)$ & $0.70(-2.3$ to 3.03$)$ & 0.57 \\
\hline$\Delta \mathrm{MDT}, \mathrm{mN}$ & $0.47(-0.97$ to 0.11$)$ & $0.02(-0.09$ to 0.26$)$ & 0.11 \\
\hline$\triangle \mathrm{MPT}, \mathrm{mN}$ & $-40.9(-71.9$ to -16$)$ & $35.5(-53.0$ to 177.4$)$ & 0.14 \\
\hline$\triangle \mathrm{MPS}, \mathrm{mN}$ & $-0.06(-0.84$ to 0.36$)$ & 0.11 ( $(-0.09$ to 0.72$)$ & 0.23 \\
\hline$\Delta W U R$, ratio & $0.03(-3.2$ to 1.3$)$ & $0.18(-1.24$ to 0.75$)$ & 0.87 \\
\hline$\Delta \mathrm{VT}$, microns & $-0.06(-0.22$ to 0.02$)$ & $-0.05(-0.13$ to 0.10$)$ & 0.52 \\
\hline$\triangle \mathrm{PPT}, \mathrm{kPa}$ & $-34.8(-115.7$ to -11.5$)$ & $-21.3(-152.7$ to 7.33$)$ & 0.66 \\
\hline
\end{tabular}

Notes: $\Delta$ indicates median preoperative value-median postoperative value. Data are medians (25-75 interquartile ranges).

Abbreviations: $\mathrm{mN}$, milliNewton; CPT, cold pain threshold; HPT, heat pain threshold; MDT, mechanical detection threshold; MPS, mechanical pain sensitivity; MPT, mechanical pain threshold; PPT, pressure pain threshold; VT, vibration threshold; WDT, warm detection threshold; WUR, wind-up ratio. 
any signs of generalized hypersensitivity. Neither of the studies included preoperative baseline values which we did.

The main limitation to this study is the relatively small sample size, but it was always meant to be a descriptive study and we believe that it is relevant as a hypothesis-generating study. It would also have been interesting to study the neuropathic, visceral, and somatic components of acute postoperative chest pain, but this was not possible because most patients would not be able to make such a subtle distinction in the acute phase.

\section{Conclusion}

We found that acute thoracic pain, but not shoulder pain or referred pain, was a risk factor for PTPS and that thoracic pain after surgery was a better predictor of chronic pain than overall pain.

\section{Acknowledgment}

The authors thank the patients who participated in the study.

\section{Author contributions}

All the authors contributed in conception and design of the study and furthermore in data analysis, data interpretation, and revising the article critically for important intellectual content. MRB-E furthermore contributed to acquisition of data and drafting the article. All the authors have approved the final version of the article and agreed to be accountable for all aspects of the work. All authors contributed toward data analysis, drafting and revising the paper and agree to be accountable for all aspects of the work.

\section{Disclosure}

The authors report no conflicts of interest in this work.

\section{References}

1. Dajczman E, Gordon A, Kreisman H, Wolkove N. Long-term postthoracotomy pain. Chest. 1991;99(2):270-274.

2. Perttunen K, Tasmuth T, Kalso E. Chronic pain after thoracic surgery: a follow-up study. Acta Anaesthesiol Scand. 1999;43(5):563-567.

3. Ochroch EA, Gottschalk A, Augostides J, et al. Long-term pain and activity during recovery from major thoracotomy using thoracic epidural analgesia. Anesthesiology. 2002;97(5):1234-1244.

4. Kehlet H, Jensen TS, Woolf CJ. Persistent postsurgical pain: risk factors and prevention. Lancet. 2006;367(9522):1618-1625.

5. Kwon ST, Zhao L, Reddy RM, et al. Evaluation of acute and chronic pain outcomes after robotic, video-assisted thoracoscopic surgery, or open anatomic pulmonary resection. J Thorac Cardiovasc Surg. 2017;154(2):652-659.e651.

6. Wildgaard K, Ravn J, Nikolajsen L, Jakobsen E, Jensen TS, Kehlet H. Consequences of persistent pain after lung cancer surgery: a nationwide questionnaire study. Acta Anaesthesiol Scand. 2011;55(1):60-68.
7. Kinney MA, Hooten WM, Cassivi SD, et al. Chronic postthoracotomy pain and health-related quality of life. Ann Thorac Surg. 2012;93(4):1242-1247.

8. Macrae WA. Chronic post-surgical pain: 10 years on. Br J Anaesth. 2008;101(1):77-86.

9. Wildgaard K, Ravn J, Kehlet H. Chronic post-thoracotomy pain: a critical review of pathogenic mechanisms and strategies for prevention. Eur J Cardiothorac Surg. 2009;36(1):170-180.

10. Bisgaard T, Rosenberg J, Kehlet H. From acute to chronic pain after laparoscopic cholecystectomy: a prospective follow-up analysis. Scand J Gastroenterol. 2005;40(11):1358-1364.

11. Callesen T, Bech K, Kehlet H. Prospective study of chronic pain after groin hernia repair. Br J Surg. 1999;86(12):1528-1531.

12. Tasmuth T, Kataja M, Blomqvist C, von Smitten K, Kalso E. Treatmentrelated factors predisposing to chronic pain in patients with breast cancer-a multivariate approach. Acta Oncol. 1997;36(6):625-630.

13. Katz J, Jackson M, Kavanagh BP, Sandler AN. Acute pain after thoracic surgery predicts long-term post-thoracotomy pain. Clin J Pain. 1996;12(1):50-55.

14. Kalso E, Perttunen K, Kaasinen S. Pain after thoracic surgery. Acta Anaesthesiol Scand. 1992;36(1):96-100.

15. Gotoda Y, Kambara N, Sakai T, Kishi Y, Kodama K, Koyama T. The morbidity, time course and predictive factors for persistent post-thoracotomy pain. Eur J Pain. 2001;5(1):89-96.

16. Pluijms WA, Steegers MA, Verhagen AF, Scheffer GJ, Wilder-Smith OH. Chronic post-thoracotomy pain: a retrospective study. Acta Anaesthesiol Scand. 2006;50(7):804-808.

17. Benedetti F, Amanzio M, Casadio C, et al. Postoperative pain and superficial abdominal reflexes after posterolateral thoracotomy. Ann Thorac Surg. 1997;64(1):207-210.

18. Benedetti F, Vighetti S, Ricco C, et al. Neurophysiologic assessment of nerve impairment in posterolateral and muscle-sparing thoracotomy. J Thorac Cardiovasc Surg. 1998;115(4):841-847.

19. Miyazaki T, Sakai T, Tsuchiya T, et al. Assessment and follow-up of intercostal nerve damage after video-assisted thoracic surgery. Eur J Cardiothorac Surg. 2011;39(6):1033-1039.

20. Rogers ML, Henderson L, Mahajan RP, Duffy JP. Preliminary findings in the neurophysiological assessment of intercostal nerve injury during thoracotomy. Eur J Cardiothorac Surg. 2002;21(2):298-301.

21. Maguire MF, Ravenscroft A, Beggs D, Duffy JP. A questionnaire study investigating the prevalence of the neuropathic component of chronic pain after thoracic surgery. Eur J Cardiothorac Surg. 2006;29(5):800-805.

22. Wildgaard K, Ringsted TK, Hansen HJ, Petersen RH, Werner MU, Kehlet $\mathrm{H}$. Quantitative sensory testing of persistent pain after video-assisted thoracic surgery lobectomy. Br J Anaesth. 2012;108(1):126-133.

23. Wildgaard K, Ringsted TK, Aasvang EK, Ravn J, Werner MU, Kehlet H. Neurophysiological characterization of persistent postthoracotomy pain. Clin J Pain. 2012;28(2):136-142.

24. Steegers MA, Snik DM, Verhagen AF, van der Drift MA, Wilder-Smith $\mathrm{OH}$. Only half of the chronic pain after thoracic surgery shows a neuropathic component. J Pain. 2008;9(10):955-961.

25. Maguire MF, Latter JA, Mahajan R, Beggs FD, Duffy JP. A study exploring the role of intercostal nerve damage in chronic pain after thoracic surgery. Eur J Cardiothorac Surg. 2006;29(6):873-879.

26. Katz J, Seltzer Z. Transition from acute to chronic postsurgical pain: risk factors and protective factors. Expert Rev Neurother. 2009;9(5):723-744.

27. Blichfeldt-Eckhardt MR, Andersen C, Ording H, Licht PB, Toft P. Shoulder pain after thoracic surgery: type and time course, a prospective cohort study. J Cardiothorac Vasc Anesth. 2017;31(1):147-151.

28. Gerner P. Postthoracotomy pain management problems. Anesthesiol Clin. 2008;26(2):355-367, vii.

29. Burgess FW, Anderson DM, Colonna D, Sborov MJ, Cavanaugh DG. Ipsilateral shoulder pain following thoracic surgery. Anesthesiology. 1993;78(2):365-368.

30. MacDougall P. Postthoracotomy shoulder pain: diagnosis and management. Curr Opin Anaesthesiol. 2008;21(1):12-15. 
31. Arendt-Nielsen L, Laursen RJ, Drewes AM. Referred pain as an indicator for neural plasticity. Prog Brain Res. 2000;129:343-356.

32. Mahtabifard A, DeArmond DT, Fuller CB, McKenna RJ Jr. Videoassisted thoracoscopic surgery lobectomy for stage I lung cancer. Thorac Surg Clin. 2007;17(2):223-231.

33. Zigmond AS, Snaith RP. The hospital anxiety and depression scale. Acta Psychiatr Scand. 1983;67(6):361-370.
34. Rolke R, Baron R, Maier C, et al. Quantitative sensory testing in the German Research Network on Neuropathic Pain (DFNS): standardized protocol and reference values. Pain. 2006;123(3):231-243.

35. Blichfeldt-Eckhardt MR, Ording H, Andersen C, Licht PB, Toft P. Early visceral pain predicts chronic pain after laparoscopic cholecystectomy. Pain. 2014;155(11):2400-2407.

\section{Publish your work in this journal}

The Journal of Pain Research is an international, peer reviewed, open access, online journal that welcomes laboratory and clinical findings in the fields of pain research and the prevention and management of pain. Original research, reviews, symposium reports, hypothesis formation and commentaries are all considered for publication.
Dovepress

The manuscript management system is completely online and includes a very quick and fair peer-review system, which is all easy to use. Visit http://www.dovepress.com/testimonials.php to read real quotes from published authors. 